\title{
Freezing a Flock: Motility-Induced Phase Separation in Polar Active Liquids
}

\author{
Delphine Geyer, ${ }^{1}$ David Martin, ${ }^{2}$ Julien Tailleur, ${ }^{2}$ and Denis Bartolo® ${ }^{1}$ \\ ${ }^{1}$ Univ. Lyon, ENS de Lyon, Univ. Claude Bernard, CNRS, Laboratoire de Physique, \\ F-69342, Lyon, France \\ ${ }^{2}$ Laboratoire Matiere et Systemes Complexes, UMR 7057 CNRS/P7, Université Paris Diderot, \\ 10 rue Alice Domon et Leonie Duquet, 75205 Paris cedex 13, France
}

(Received 27 February 2019; revised manuscript received 3 June 2019; published 9 September 2019)

\begin{abstract}
Combining model experiments and theory, we investigate the dense phases of polar active matter beyond the conventional flocking picture. We show that above a critical density flocks assembled from selfpropelled colloids arrest their collective motion, lose their orientational order, and form solids that actively rearrange their local structure while continuously melting and freezing at their boundaries. We establish that active solidification is a first-order dynamical transition: active solids nucleate, grow, and slowly coarsen until complete phase separation with the polar liquids with which they coexist. We then theoretically elucidate this phase behavior by introducing a minimal hydrodynamic description of dense polar flocks and show that the active solids originate from a motility-induced phase separation. We argue that the suppression of collective motion in the form of solid jams is a generic feature of flocks assembled from motile units that reduce their speed as density increases, a feature common to a broad class of active bodies, from synthetic colloids to living creatures.
\end{abstract}

DOI: 10.1103/PhysRevX.9.031043

\section{INTRODUCTION}

The emergence of collective motion in groups of living creatures or synthetic motile units is now a well-established physical process [1-6]: Self-propelled particles move coherently along the same direction whenever velocityalignment interactions overcome orientational perturbations favoring isotropic random motion. This minimal picture goes back to the seminal work of Vicsek et al. [7] and made it possible to elucidate the flocking dynamics of systems as diverse as bird groups, polymers surfing on motility assays, shaken grains, active colloidal fluids, and drone fleets [6,8-13]. From a theoretical perspective, flocks are described as flying ferromagnets where pointwise spins move at constant speed along their spin direction $[1,3,7,14,15]$. However, this simplified description fails to capture the dynamics of dense populations where contact interactions interfere with self-propulsion and ultimately arrest the particle dynamics. Until now, aside from rare theoretical exceptions [14,16-19], the consequences of motility reduction in dense flocks has remained virtually uncharted despite its relevance to a spectrum of active

Published by the American Physical Society under the terms of the Creative Commons Attribution 4.0 International license. Further distribution of this work must maintain attribution to the author(s) and the published article's title, journal citation, and DOI.
Subject Areas: Fluid Dynamics, Soft Matter,

Statistical Physics bodies ranging from marching animals to robot fleets and active colloids.

In this article, combining quantitative experiments and theory, we investigate the suppression of collective motion in high-density flocks. We show and explain how polar assemblies of motile colloids turn into lively solid phases that actively rearrange their amorphous structure but do not support any directed motion. We establish that active solidification of polar liquids is a first-order dynamical transition: active solids nucleate, grow, and slowly coarsen until complete phase separation. Even though they are mostly formed of particles at rest, we show that active solids steadily propagate through the polar liquids with which they coexist. Using numerical simulations and analytical theory, we elucidate all our experimental findings and demonstrate that the solidification of colloidal flocks provides a realization of the long-sought-after complete motility-induced phase separation (MIPS) [5,20-28].

\section{SOLIDIFICATION OF COLLOIDAL FLOCKS}

Our experiments are based on the colloidal-roller system introduced in Ref. [10]. In short, we let polystyrene colloids sediment on a flat electrode (radius $a=2.4 \mu \mathrm{m}$ ). Applying a dc electric field $\mathbf{E}_{\mathbf{0}}$ normal to the solid surface turns the inert beads into self-propelled rollers. The particles then all move at the same constant speed $\nu_{0}=1.05 \mathrm{~mm} / \mathrm{s}$ when isolated, while their rolling directions undergo rotational diffusion with a rotational diffusivity of the order of $6.0 \mathrm{~s}^{-1}$. 

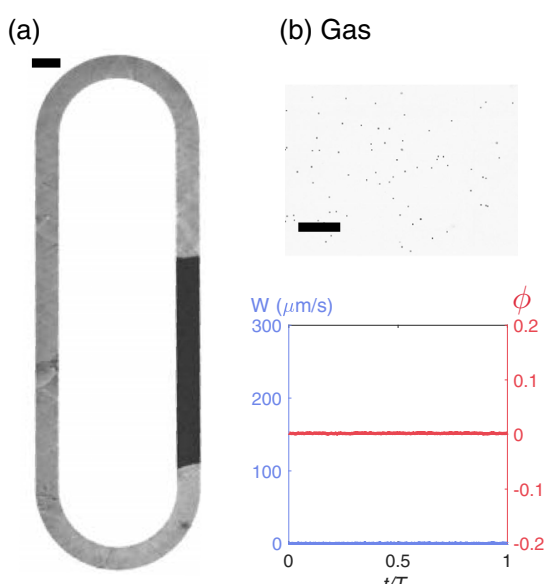

(c) Vicsek bands

(d) Polar liquid

(e) Solid jam

(f) Active solid
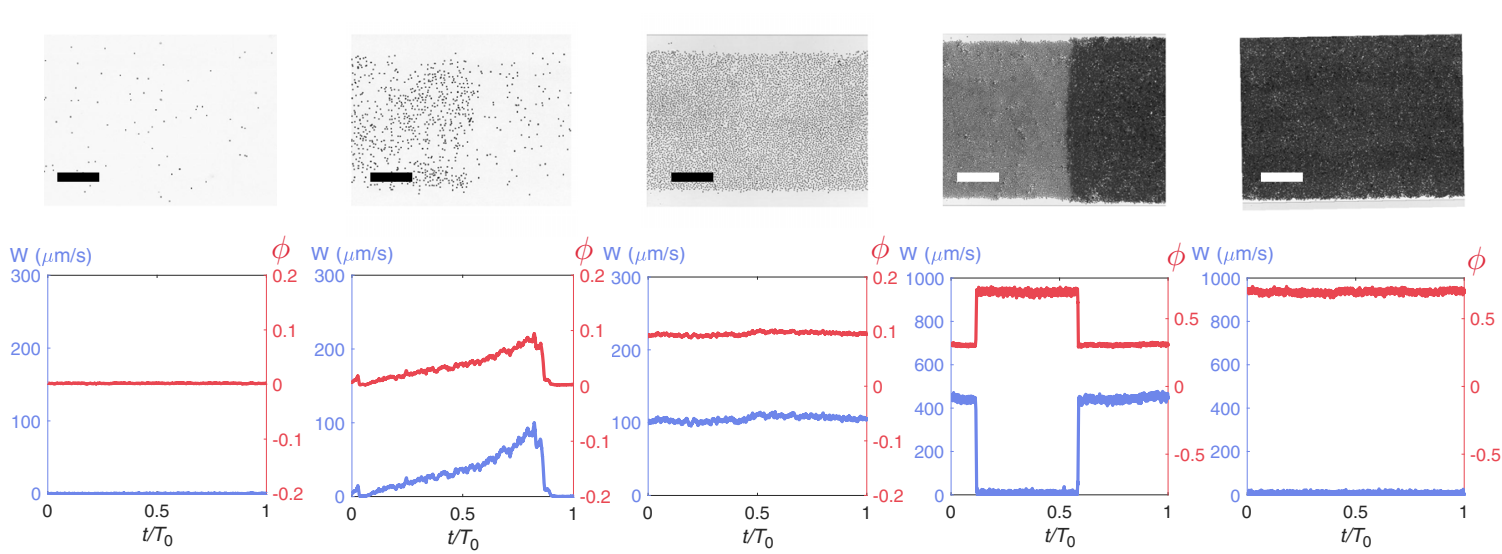

FIG. 1. The dynamical phases of Quincke rollers. (a) Picture of a microfluidic racetrack where $\sim 3 \times 10^{6}$ Quincke rollers interact. An active solid (dark gray) propagates through a polar liquid (light gray). Scale bar is $2 \mathrm{~mm}$. (b)-(f) Top: Close-up pictures of Quincke rollers in the racetrack. Scale bars are $250 \mu \mathrm{m}$. Bottom: Longitudinal component of the particle current $W$ and density plotted as a function of a normalized time. Both $W$ and $\phi$ are averaged over an observation window of size $56 \times 1120 \mu \mathrm{m}^{2} . T_{0}$ is arbitrarily chosen to be the time taken by an active solid to circle around the race track. (b) Gas phase $\left(\phi_{0}=0.002\right)$. The density is homogeneous and the system does not support any net particle current. (c) Coexistence between an active gas and a denser polar band $\left(\phi_{0}=0.033\right)$. A heterogeneous polar-liquid drop propagates at constant speed through a homogeneous isotropic gas in the form of a so-called Vicsek band. (d) Polar-liquid phase $\left(\phi_{0}=0.096\right)$. The homogeneous active fluid supports a net flow. (e) Coexistence between a polar liquid and an amorphous active solid $\left(\phi_{0}=0.49\right)$. The high-density solid is homogeneous but, unlike the polar liquid, does not support any net particle current. (f) Homogeneous active-solid phase $\left(\phi_{0}=0.70\right)$.

The motorization principle relies on the so-called Quincke electrorotation phenomenon [29], which we recall in the Appendix A. We observe the colloidal rollers propelling and interacting in microfluidic racetracks of length $L_{0}=9.8 \mathrm{~cm}$ and width $2 \mathrm{~mm}$; see Fig. 1(a). Both hydrodynamic and electrostatic interactions promote alignment of the roller velocities. As a result, the low-density phase behavior of the Quincke rollers falls in the universality class of the Vicsek model [7,30-32]. At low density, Fig. 1(b), they form an isotropic gas where the packing fraction $\phi(\mathbf{r}, t)$ is homogeneous and the local particle current $W(\mathbf{r}, t)$ vanishes. Increasing the average packing fraction $\phi_{0}$ above 0.02, the rollers undergo a flocking transition; see Fig. 1(c). The transition is first order, and polar liquid bands, where all colloids propel on average along the same direction, coexist with an isotropic gas [10]. Further increasing $\phi_{0}$, the ordered phase fills the entire system and forms a homogeneous polar liquid which flows steadily and uniformly, as illustrated in Movie 1 of Supplemental Material [33]. In polar liquids, both $\mathbf{W}(\mathbf{r}, t)$ and $\phi(\mathbf{r}, t)$ display small (yet anomalous) fluctuations, and orientational order almost saturates; see Fig. 1(d) and Ref. [34]. This low-density behavior provides a prototypical example of flocking physics.

However, when $\phi_{0}$ exceeds $\phi_{S} \simeq 0.55$, collective motion is locally suppressed and flocking physics fails in explaining our experimental observations. Particles stop their collective motion and jam, as exemplified in Movies 2 and 3 of Supplemental Material [33]. The jams are active solids that continuously melt at one end while growing at the other end. This lively dynamics hence preserves the shape and length $\left(L_{S}\right)$ of the solid which propagates at constant speed upstream the polar-liquid flow; see the kymograph of Fig. 2(a). Further increasing $\phi_{0}$, the solid region grows and eventually spans the entire system; see Fig. 1(f).

Active solids form an amorphous phase. The paircorrelation function shown in Figs. 2(b) and 2(c) indicates that active solids are more spatially ordered than the polar liquid with which they coexist, but do not display any sign of long-range translational order. We note that the location of the first peak of $g(r)$ drops from a value that is larger than a colloid diameter in the liquid to one particle diameter in the solid phase. This noticeable feature distinguishes the solidification process from that reported in Ref. [35] for self-propelled granular hard disks. The large value of the typical interparticle distance in the densest liquid phase reflects the repulsive dipolar interactions acting on neighboring Quincke rollers [10,36-38]. But the most striking difference between the two phases is dynamical. As clearly seen in Movie 2 of Supplemental Material [33], the rollers continuously move at constant speed in the polar liquid. In contrast, in the solid phase, they spend most of their time at rest, thereby preventing the existence of long-range orientational order in their velocities; see Figs. 2(d) and 2(e).

We stress that the onset of active solidification corresponds to an area fraction $\phi_{S} \simeq 0.55$, which is much smaller than the random close-packing limit $\left(\phi_{0}=0.84\right)$ and than the crystallization point of self-propelled hard disks $\left(\phi_{0} \sim 0.71\right)$ reported in Ref. [35]. This marked difference hints towards different physics, which we characterize and elucidate below. 



FIG. 2. Structure and dynamics of active jams. (a) The kymograph of the measured light intensity averaged over the racetrack width shows how an active solid (dark region) propagates at constant speed through a homogeneous polar liquid (light region). (b),(c) Pair-correlation functions measured in the polar liquid $\left(g_{L}\right)$ and in the coexisting active-solid phase $\left(g_{S}\right)$. $\phi_{0}=0.58$. Both pair-correlation functions are plotted versus the interparticle distance $r_{\perp}$ in the direction transverse to the mean polar-liquid flow. $g_{S}$ displays more peaks than $g_{L}$, revealing a more ordered structure, but translational order merely persists over a few particle diameters. The dashed lines indicate the distance corresponding to one particle diameter. (d) Probability density functions of the roller velocities in the polar-liquid region. The distribution is peaked around $\nu_{0} \hat{\mathbf{x}}_{\|}$, where $\hat{\mathbf{x}}_{\|}$is the vector tangent to the racetrack centerline. (e) Probability density functions of the roller velocities in the active jam region. The distribution is peaked around 0 . The rollers remain mostly at rest. (b)-(e) Average area fraction $\phi_{0}=0.58$.

\section{EMERGENCE OF AMORPHOUS ACTIVE SOLIDS IS A FIRST-ORDER PHASE SEPARATION}

We now establish that the formation of active solids occurs according to a first-order phase-separation scenario. Firstly, Figs. 3(a) and 3(b) indicate that, upon increasing $\phi_{0}$, the extent of the solid phase has a lower bound: starting from a homogeneous polar liquid, the solid length $L_{S}$ discontinuously jumps to a finite value before increasing linearly with $\phi_{0}-\phi_{L}^{b}$, where $\phi_{L}^{b}=0.55$; see Fig. 3(b). The smallest solid observed in a stationary state is as large as $L_{S} \sim 1.4 \mathrm{~cm}$. Smaller transient solid jams do form at local heterogeneities, but they merely propagate over a finite distance before rapidly melting; see Movie 4 in Supplemental Material [33]. This observation suggests the existence of a critical nucleation radius for the solid phase. Although the phenomenology is preserved, we note that the minimal fraction for the formation of an active solid is larger than that reported in Fig. 1 as we used two different formulations of the colloid batch; see Appendix B 1. We henceforth report measurements all performed under the same experimental conditions.

Secondly, while the packing fraction of a polar liquid obviously increases with $\phi_{0}$, its value saturates as it coexists with an active solid; see Fig. 3(c). At coexistence, the local packing fractions in the bulk of the liquid and solid phases are independent of the average fraction $\phi_{0}, \phi_{S}^{b}=$ 0.75 and $\phi_{L}^{b}=0.55$, which again supports a nucleation and growth scenario. (Note that $\phi_{L}^{b}$ is hardly distinguishable from the packing fraction at the onset of solidification $\phi_{S}$ in our experiments, because the system size is much larger than the size of the nucleated active solids.) Increasing $\phi_{0}$ leaves the inner structure of both phases unchanged and solely increases the fraction of solid $L_{S} / L_{0}$ in the racetrack. We find that, as in equilibrium phase separation, the length of the solid region is accurately predicted using a lever rule constructed from the stationary bulk densities $\phi_{S}^{b}$ and $\phi_{L}^{b}$; see Fig. 3(b).

Thirdly, we stress that when multiple jams nucleate in the device, they propagate nearly at the same speed; see Movie 4 of Supplemental Material [33]. Therefore, they cannot catch up and coalesce. The system in fact reaches a stationary state thanks to a slow coarsening dynamics illustrated in Fig. 3(d), where we show the temporal evolution of the length of two macroscopic active solids (red symbols) and of the overall solid fraction (dark line). This experiment corresponds to a situation where the smallest solid jam grows at the expense of the larger. The converse situation is also possible (see Movie 4 of Supplemental Material [33]). In both cases coarsening operates, leaving the overall fraction of solid constant. All of our experiments end with complete phase separation: a single macroscopic active solid coexists with a single active liquid phase. The final state of the system is therefore uniquely determined by two macroscopic control parameters: the average packing fraction $\phi_{0}$ and the magnitude $E_{0}$ of the electric field used to power the rollers.

Finally, the most compelling argument in favor of a genuine first-order phase separation is the bistability of the two phases. Figure 3(b) shows that at the onset of solidification, depending on the (uncontrolled) initial conditions, the system is observed either in a homogeneous polar liquid or at liquid-solid coexistence. The bistability of the active material is even better evidenced when cycling the magnitude $E_{0}$ of the electric field that powers the rollers' motion (cycling the average density is not experimentally feasible). Figure 3(e) shows the temporal variations of the active-solid fraction upon triangular modulation of $E_{0}$; see also Movie 5 of Supplemental Material [33]. When $E_{0}$ increases, an active-solid nucleates and quickly 
(a)

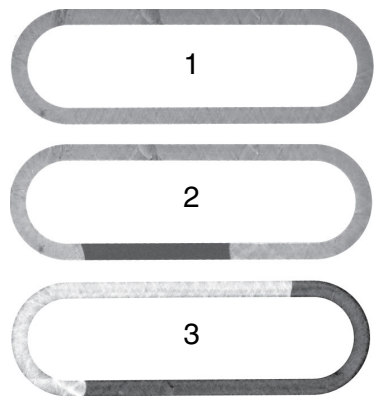

(d)

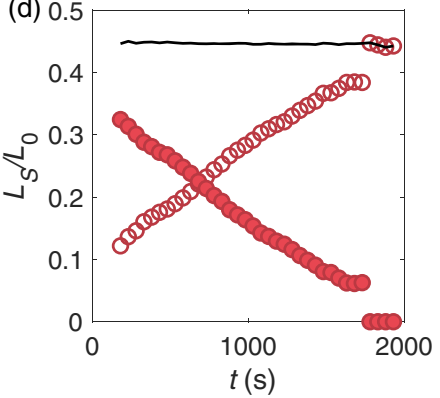

(b)

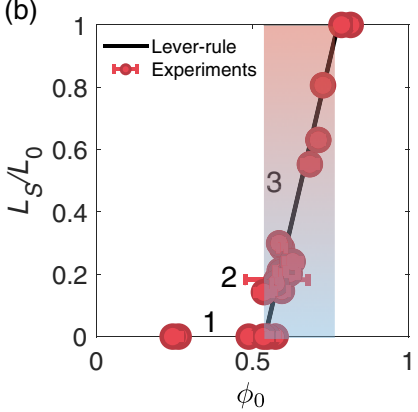

(e)

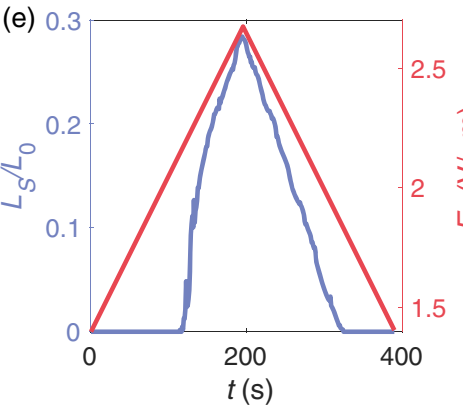

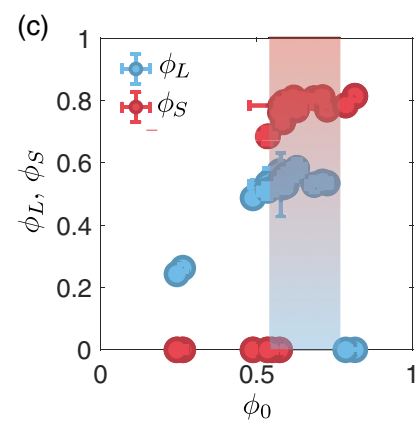

(f)

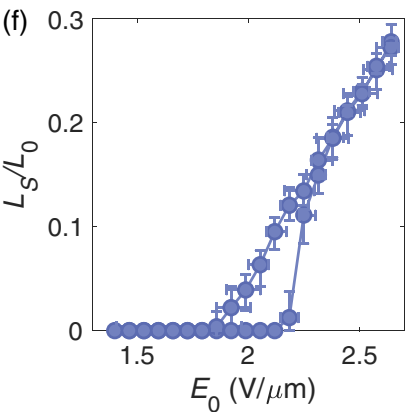

FIG. 3. Active solidification is a first-order phase separation. (a) Solid jams in a racetrack at $\phi_{0}=0.38,0.58,0.65$. Increasing the average packing fraction, the extent of the active solid increases. (b) Solid fraction plotted versus the average packing fraction $\phi_{0}$. Note the discontinuous jump and the two possible states at the onset of solidification. (c) Density of the polar-liquid phase (blue circles) and of the active-solid phase (red circles) plotted versus $\phi_{0}$. In steady state, the liquid density increases with $\phi_{0}$ until an active solid forms, the density in both phases then remains constant. In (b) and (c) the shaded regions indicate the coexistence between the polar-liquid and active-solid phases. (d) Coarsening dynamics. Two solid jams coexist only over a finite time period. The larger jam (filled symbols) shrinks and eventually vanishes in favor of the smaller one (open symbols). The total fraction of solid phase in the racetrack (black line) remains constant over time. (e) Solid fraction (blue line) and magnitude of the electric field (red line) plotted versus time. Over a range of $E_{0}$ values, the active-solid fraction is different when increasing or decreasing the electric field. (f) The extent of the traffic jam follows a hysteresis loop when cycling the field amplitude.

grows. When $E_{0}$ decreases, the solid continuously shrinks and eventually vanishes at a field value smaller than the nucleation point. Note that varying $E_{0}$ changes the speed of the particles as well as their interactions. These microscopic changes explain the variations of the packing fractions of the two coexisting phases, which in turn account for the variation of the domain sizes at fixed average density. The asymmetric dynamics of $L_{S} / L_{0}$ demonstrates the existence of a metastable region in the phase diagram. As shown in Fig. 3(f), the metastability of the active solid results in the hysteretic response of $L_{S}$, the hallmark of a first-order phase transition. We also note that the continuous interfacial melting observed when $E_{0}$ smoothly decreases contrasts with the response to a rapid field quench; see Movie 6 of Supplemental Material [33]. Starting with a stationary active solid, a rapid quench deep in the coexistence region results in a destabilization of the solid bulk akin to a spinodal decomposition dynamics. Finally, as illustrated in Movie 7 of Supplemental Material [33], when repeating the same experiments in isotropic 6-mm-wide circular chambers, we observe the same macroscopic phase separation into active solids and polar liquids. This observation confirms that solidification is a bulk phenomenon which does not rely on specific geometrical parameters.
Altogether these measurements and observations establish that the emergence of active solids results from a first-order phase separation, which we theoretically elucidate below.

\section{MOTILITY-INDUCED PHASE SEPARATION IN HIGH-DENSITY POLAR FLOCKS}

\section{A. Nonlinear hydrodynamic theory}

As a last experimental result, we show in Fig. 4(a) how the roller speed $\nu_{0}(\phi)$ varies with the local density $\phi(\mathbf{r}, t)$ evaluated in square regions of size $12 a \sim 29 \mu \mathrm{m}$. These measurements correspond to an experiment where a solid jam coexists with a homogeneous polar liquid. $\nu_{0}(\phi)$ hardly varies at the smallest densities and sharply drops towards $\nu_{0}(\phi)=0$ when the local fraction $\phi(\mathbf{r}, t)$ exceeds $\sim 0.35$. Although we cannot positively identify the microscopic origin of this abrupt slowing-down, we detail a possible explanation in Appendix A. Simply put, the lubrication interactions between nearby colloids with collinear polarizations result in the reduction of their rotation rate, which ultimately vanishes at contact: near-field hydrodynamic interactions frustrate self-propulsion. Instead of elaborating a microscopic theory specific to colloidal rollers as in Ref. [10], we instead adopt a generic hydrodynamic description to account for all our experimental findings. 

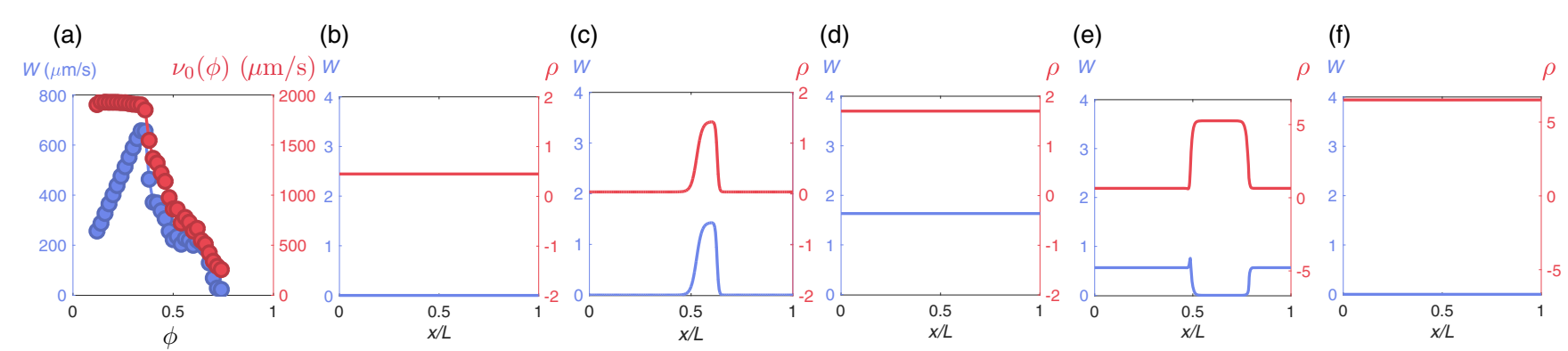

FIG. 4. Nonlinear hydrodynamics of polar active matter. (a) Average velocity $\nu_{0}(\mathbf{r}, t)$ of the colloids and local magnitude of the longitudinal current $W(\mathbf{r}, t)$ plotted as a function of the local packing fraction $\phi(\mathbf{r}, t)$. (b)-(e) Successive phases observed in the resolution of Eqs. (1) and (2) at increasing average densities $\rho_{0}=(0.10,0.18,1.7,1.96,6.5)$. The position $x$ is normalized by the system size $L$. Simulation parameters are $D_{\rho}=0.2, D_{W}=1, \lambda=1, a_{4}=0.45, \sigma=0.2, \bar{\rho}=2, \xi=0.01, \alpha=1, \rho_{c}=0.5, L=200$, $d x=0.05, d t=0.005$.

We start with a minimal version of the Toner-Tu equations which proved to correctly capture the coexistence of active gas and polar-liquid drops at the onset of collective motion $[2,39,40]$. For the sake of simplicity we ignore fluctuations transverse to the mean-flow direction and write the hydrodynamic equations for the one-dimensional longitudinal current $W(x, t)$ and number-density field $\rho(x, t)$ :

$$
\begin{gathered}
\partial_{t} \rho+\partial_{x} W=D_{\rho} \partial_{x x} \rho \\
\partial_{t} W+\lambda W \partial_{x} W=D_{W} \partial_{x x} W-\partial_{x}[p(\rho)] \\
+a_{2}(\rho) W-a_{4} W^{3},
\end{gathered}
$$

where $D_{\rho}, \lambda, D_{W}, a_{4}$ are constant hydrodynamic coefficients. $a_{2}(\rho)$ is a function of $\rho$ that changes sign and becomes negative as $\rho$ exceeds a critical value $\rho_{c}$, thereby allowing for a flocking transition upon increasing the particle density [39,40], and $p(\rho)$ is the so-called pressure term [2]. Interestingly, the slowing-down of the rollers at high density, Fig. 4(a), can be accounted for through specific choices of $a_{2}(\rho)$ and $p(\rho)$, which we detail below.

Firstly, coarse graining microscopic flocking models typically leads to a pressure term proportional to the particle speed $[1,14,31]$. We therefore expect $p(\rho)$ to sharply decrease when $\rho(x, t)>\bar{\rho}$. Secondly, $a_{2}(\rho)$ is also expected to decay and change sign, given the loss of the orientational order of the particle velocities in the solid phase reported in Fig. 2(e). As the roller speed and the suppression of orientational order happen concomitantly, in all that follows, we conveniently choose $a_{2}(\rho)=$ $\alpha\left[\rho \epsilon(\rho)-\rho_{c}\right]$ and $p(\rho)=\sigma \rho \epsilon(\rho)$, where the function $\epsilon(\rho)$ decreases from 1 in the low-density phases to a vanishing value deep in the solid phase, and where $\alpha$ and $\sigma$ are two positive constants. In practice, we take $\epsilon(\rho)=\{1-\tanh [(\rho-\bar{\rho}) / \xi]\} / 2$. We note that Eqs. (1) and (2) and the functional form of $\epsilon(\rho)$ are here postulated on phenomenological grounds. They could alternatively be constructed from the explicit coarse graining of microscopic models using well-established methods and approximations
$[14,31,41,42]$. The precise form of the decay of $a_{2}(\rho)$ and $p(\rho)$ would then depend on the specifics of the microscopic dynamics, but would leave our conclusions qualitatively unchanged $[32,39,40]$. Finally, at densities larger than $\bar{\rho}$, we also expect the repulsion and contact interactions between the particles to result in a pressure increase with the particle density $[10,41]$. We henceforth disregard this second regime, which is not essential to the nucleation and propagation of active solids.

Numerical resolutions of Eqs. (1) and (2) at increasing densities faithfully account for the five successive phases observed in our experiments; see Fig. 4 and Appendix B. At low densities, we first observe the standard Vicsek transition: a disordered gas phase is separated from a homogeneous polar-liquid phase by a coexistence region where ordered bands propagate through a disordered background [15]. This phase transition occurs at very low densities $\left(\rho_{0} \sim \rho_{c} \ll \bar{\rho}\right)$, in a regime where the colloidal rollers experience no form of kinetic hindrance as they interact; therefore, $\epsilon(\rho) \simeq 1$. In agreement with our experiments, a second transition leads to the coexistence between a polar liquid of constant density $\rho_{L}^{b}$ and an apolar dense phase of constant density $\rho_{S}^{b}$. This jammed phase propagates backwards with respect to the flow of the polar liquid as does the active solids we observe in our experiments. This second transition shares all the signatures of the first-order phase separation reported in Fig. 3. Figures 5(a)5(c) indicate that the jammed phase obeys a lever rule-its width increases linearly with $\rho_{0}-\rho_{L}^{b}$-while its velocity $c$ and the shape of the fronts remain unchanged upon increasing $\rho_{0}$. The first-order nature of the transition is further supported by Movie 8 of Supplemental Material [33], which shows the existence of a hysteresis loop when ramping up and down the average density.

\section{B. Spinodal instability of polar liquids and domain wall propagation}

Having established the predictive power of our hydrodynamic model, we now use it to gain physical insight into the origin of active solidification. We focus on the 


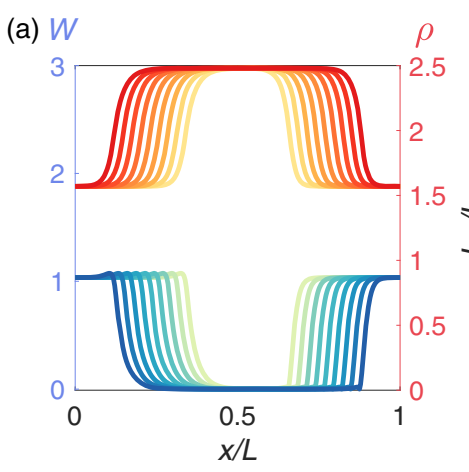

(b)

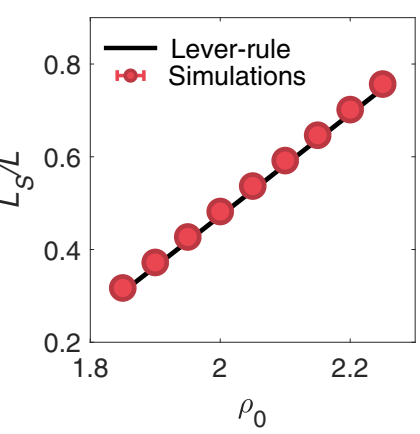

(c)

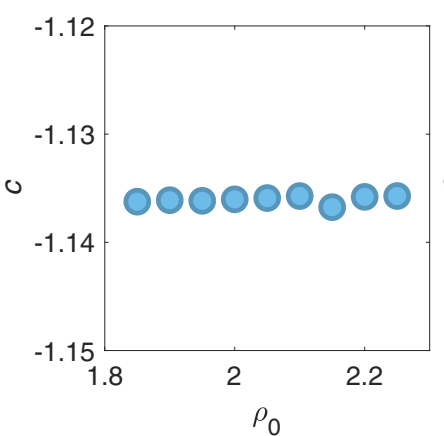

(d)

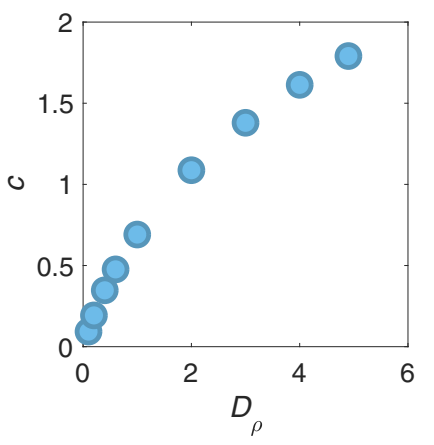

FIG. 5. Shape and dynamics of active-solid jams. (a) Density and velocity profiles computed for different value of $\rho_{0}$ : from 1.85 (light colors) to 2.25 (dark colors). Numerical resolution of Eqs. (1) and (2) with the hydrodynamic parameters $D_{\rho}=5, D_{W}=10, \lambda=1$, $a_{4}=1, \sigma=1, \bar{\rho}=2, \xi=0.1, \alpha=1, \rho_{c}=0.5, L=200, d x=0.01, d t=0.001$. (b) Length of the solid jam plotted versus $\rho_{0}$ (symbols) and lever rule (solid line). Same parameters as in (a). (c) The speed of the solid jam $c$ does not depend on the average density $\rho_{0}$. (d) Variations of the propagation speed $c$ as a function of the effective diffusivity $D_{\rho}$. Numerical parameters are $D_{W}=5, x i=1$, $a_{4}=0.45, \sigma=0.2, \bar{\rho}=2, \xi=0.01, \alpha=1, \rho_{c}=0.5, L=200, d x=0.05, d t=0.005, \rho_{0}=1.96$.

experimentally relevant situation where the slowing-down of the particle occurs at area fractions much larger than that of the onset of collective motion. Given this hierarchy, at low densities, when $\rho_{0} \ll \bar{\rho}$, the hydrodynamic equations Eqs. (1) and (2) correspond to that thoroughly studied in Refs. [39,40,43]. They correctly predict a first-order transition from an isotropic gas to a polar-liquid phase; see also Appendix C.

The phase separation between a polar liquid and a jammed phase also becomes clear when performing a linear stability analysis of the homogeneous solutions of Eqs. (1) and (2); see Appendix C where the stability of the various phases is carefully discussed. At high density, the stability of polar liquids where $\rho=\rho_{0}$ and $W=W_{0} \neq 0$ is limited by a phenomenon that is not captured by classical flocking models. When $\rho_{0} \gg \rho_{c}+\epsilon_{2} /\left(2 \lambda \epsilon_{2}+4 a_{4} \epsilon_{1}\right)$, polar liquids are stable with respect to the spinodal decomposition into Vicsek bands; however, another instability sets in whenever $\epsilon^{\prime}\left(\rho_{0}\right) \rho_{0}+\epsilon\left(\rho_{0}\right)<-W_{0}^{2} K$, with $K$ a positive constant given in Appendix C. This condition is met when $\rho$ approaches $\bar{\rho}$, thereby leading to the formation of active-solid jams. We learn from the stability analysis that it ultimately relies on the decrease of the effective pressure with density in Eq. (2) as a result of the slowing-down of the colloids in dense environments. This criterion is exactly analogous to the spinodal decomposition condition in MIPS physics: the formation of active-solid jams results from a complete motility-induced phase separation [20].

\section{Discussion}

Three comments are in order. Firstly, our results provide a novel microscopic mechanism leading to MIPS. In classical systems such as active Brownian particles, repulsive interactions and persistent motion conspire to reduce the local current when active particles undergo head-on collisions $[21,23,24]$. Here we show that this microscopic dynamics is, however, not necessary to observe phase separation, and MIPS transitions solely rely on the reduction of the active particle current as density becomes sufficiently high, irrespective of its microscopic origin. In the case of colloidal rollers, particles indeed do not experience any frontal collision when an active solid nucleates in a polar liquid; phase separation is, however, made possible by the slowing-down of their rolling motion.

Secondly, the coarsening dynamics clearly differ from that reported for MIPS between a disordered gas and an ordered liquid [23,26,27,44-46]. This is not unexpected given the previous results on the coarsening of flocking patterns in slender geometries [40,41], and the absence of the very concept of surface tension, which remains to be elucidated in active fluids.

Finally, another marked difference with the dense phases of conventional MIPS system is the steady propagation of the active solids through the dilute polar liquid. This dynamics can be accounted for by our model. The two boundaries of the active solid are two domain walls that propagate at the same speed. The propagation of the domain wall at the front of the solid jam relies on a mechanism akin to actual traffic jam propagation: the directed motion of the particles incoming from the polar liquid causes an accumulation at the boundary with the arrested phase, in the direction opposing the spontaneous flow. By contrast, the propagation of the second domain wall, at the back of the solid jam, requires arrested particles to resume their motion. The formation of this smooth front originates from the mass diffusion terms in Eq. (1), which allows particles to escape the arrested solid phase and progressively resume their collective motion when reaching a region of sufficiently low density in the polar liquid. This diffusive spreading, however, does not rely on thermal diffusion. The roller diffusivity, $D_{m} \sim 10^{-13} \mathrm{~m}^{2} / \mathrm{s}$, is indeed 
negligible on the timescale of the experiments. Fortunately, other microscopic mechanisms, and in particular anisotropic interactions, lead to diffusive contributions to the density current [47]. A simple way to model this effect is to consider a velocity-density relation of the form $\nu_{0}(\rho)[1-r \mathbf{u} \cdot \nabla \rho]$, where $\mathbf{u}$ is the orientation of the particle and $r$, which could be density dependent, quantifies the anisotropic slowingdown of the particles which ascend density gradients. This anisotropic form is consistent with the polar symmetry of the flow and electric field induced by the Quincke rotation of the colloids. Coarse graining the dynamics of self-propelled particles interacting via such a nonlocal quorum-sensing rule was done in Ref. [47] and leads to an effective Fickian contribution, $\sim-\rho \nu_{0} r \nabla \rho$, to the density current in Eq. (1). The ratio between the magnitude of this effective Fickian flux and that of thermal diffusion is readily estimated as $\left(\rho \nu_{0} r\right) / D_{m} \sim 10^{5}$, assuming that $r$ is of the order of the colloid diameter. Anisotropic interactions are therefore expected to strongly amplify the magnitude of $D_{\rho}$. In order to confirm the prominent role of this diffusion term in the active-solid dynamics, we numerically measure the propagation speed $c$ of the jammed region as a function of $D_{\rho}$. In agreement with the above discussion, $c$ is found to vanish as $D_{\rho} \rightarrow 0$, Fig. 5(d), thereby confirming the requirement of a finite diffusivity to observe stable active-solid jams. Simply put, the steady propagation of active solids relies on the balance between two distinct macroscopic phenomena: motility reduction at high density, which results in the formation of sharp interfaces with the polar liquid, and the diffusive smoothing of the interfaces that enables particles trapped in the arrested solid phase to resume their motion by rejoining the polar-liquid flock.

\section{CONCLUSION}

In summary, combining experiments on Quincke rollers and active-matter theory, we show that the phase behavior of polar active units is controlled by a series of two dynamical transitions: a Flocking transition that transforms active gases into spontaneously flowing liquids and a motility-induced phase separation that results in the freezing of these polar fluids and the formation of active solids. Although most of their constituents are immobile, activesolid jams steadily propagate through the active liquid with which they coexist due to their continuous melting and freezing at their boundaries. Remarkably, Quincke rollers provide a rare example of an unhindered MIPS dynamics that is not bound to form only finite-size clusters; see Ref. [48], and references therein. Beyond the specifics of active rollers, we show that the freezing of flocking motion and the emergence of active solids is a generic feature that solely relies on polar ordering and speed reduction in dense environments. A natural question to ask is wether suitably tailored polar and quorum-sensing interactions could yield ordered active solids. More generally understanding the inner structure and dynamic of active solids is an open challenge to active matter physicists.

\section{ACKNOWLEDGMENTS}

We thank A. Morin and A. Chardac for invaluable help with the experiments.

D. G. and D. M. contributed equally to this work.

\section{APPENDIX A: QUINCKE ROLLERS}

\section{Motorization}

Our experiments are based on colloidal rollers [10]. We motorize inert polystyrene colloids of radius $a=2.4 \mu \mathrm{m}$ by taking advantage of the so-called Quincke electrorotation instability [29,49]. Applying a dc electric field to an insulating body immersed in a conducting fluid results in a surface-charge dipole $\mathbf{P}$. Increasing the magnitude of the electric field $\mathbf{E}_{0}$ above the Quincke threshold $E_{Q}$ destabilizes the dipole orientation, which in turn makes a finite angle with the electric field. A net electric torque $\mathbf{T}_{E} \sim \mathbf{P} \times \mathbf{E}_{0}$ builds up and competes with a viscous frictional torque $\mathbf{T}_{V} \sim \eta \boldsymbol{\Omega}$, where $\boldsymbol{\Omega}$ is the colloid rotation rate and $\eta$ is the fluid shear viscosity. In steady state, the two torques balance and the colloids rotate at constant angular velocity. As sketched in Figs. 6(a) and 6(b), when the colloids are let to sediment on a flat electrode, rotation is readily converted into translational motion at constant speed $v_{0}$ (in the direction opposite to the charge dipole). We stress that the direction of motion is randomly chosen and freely diffuses as a result of the spontaneous symmetry breaking of the surface-charge distribution. Within our experimental conditions the rotational diffusivity of the Quincke rollers measured from the decay of the velocity autocorrelation function in the gas phase is $6 \mathrm{~s}^{-1}[36,38]$.

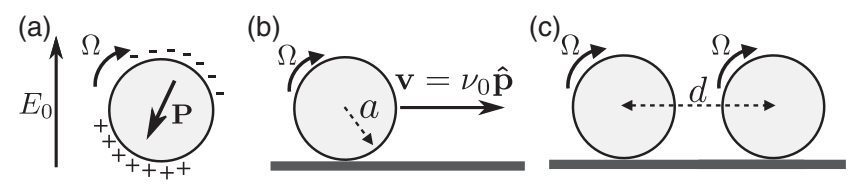

FIG. 6. Quincke rollers. (a) When applying a dc electric field $\mathbf{E}_{\mathbf{0}}$ to an insulating sphere immersed in a conducting fluid, a charge dipole forms at the sphere surface. When $E_{0}>E_{Q}$, the electric dipole makes a finite angle with the electric field causing the steady rotation of the sphere at constant angular speed $\Omega$. (b) The rotation is converted into translation by allowing the sphere to sediment on one electrode. When isolated, the resulting Quincke rotor rolls without sliding at constant speed: $\nu_{0}(0)=$ $a \Omega$. (c) When two colloids rolling in the same direction are close to each other, the lubrication torque acting on the two spheres separated by a distance $d$ scales as $\log (d-2 a)$ and hinders their rolling motion. 


\section{Arresting Quincke rotation}

We conjecture a possible microscopic mechanism to explain the arrest of the Quincke rotation at high area fraction: the frustration of rolling motion by lubrication interactions; see Fig. 6(c). The viscous torque $\mathbf{T}_{V}$ acting on two nearby colloids rolling along the same direction is chiefly set by the lubricated flow in the contact region separating the two spheres. $T_{V}$ therefore increases logarithmically with $d-2 a$, where $d$ is the interparticle distance [50]. As there exists an upper bound to the magnitude of the electric torque $T_{E}$, torque balance requires the rolling motion to become vanishingly slow as $d-2 a$ goes to zero: lubricated contacts frustrate collective motion.

\section{APPENDIX B: EXPERIMENTAL AND NUMERICAL METHODS}

\section{Experiments}

The experimental setup is identical to that described in Ref. [34]. We disperse polystyrene colloids of radius $a=$ $2.4 \mu \mathrm{m}$ (Thermo Scientific G0500) in a solution of hexadecane including $0.055 \mathrm{wt} \%$ of AOT(Dioctyl sulfosuccinate sodium salt) salt (only the data reported in Fig. 1 correspond to an AOT concentration of $0.040 \mathrm{wt} \%$ leaving the global phenomenology modified but changing the values of the microscopic interaction parameters). We then inject the solution in microfluidic chambers made of two electrodes spaced by a $110-\mu$ m-thick Scotch tape. The electrodes are glass slides, coated with indium tin oxide (Solems, ITOSOL30, thickness $80 \mathrm{~nm}$ ). We apply a dc electric field between the two electrodes ranging from 1.3 to $2.6 \mathrm{~V} / \mu \mathrm{m}$ using a voltage amplifier. If not specified otherwise, the data correspond to experiments performed at $1.8 \mathrm{~V} / \mu \mathrm{m}$, i.e., at $E_{0} / E_{Q}=2$. We confine the rollers inside racetracks by coating the bottom electrode with an insulating pattern preventing the electric current from flowing outside of the racetrack. To do so, we apply a $2-\mu \mathrm{m}$-thick layer of insulating photoresist resin (Microposit S1818) and pattern it by means of conventional UV lithography, as explained in Ref. [34].

In order to keep track of the individual colloid position and velocity, we image the system with a Nikon AZ100 microscope with a $4.8 \times$ magnification and record videos with a CMOS camera (Basler Ace) at a frame rate up to $900 \mathrm{~Hz}$. We use conventional techniques to detect and track all particles [51-53]. When performing large-scale observations, we use a different setup composed of a $60-\mathrm{mm}$ macro lens (Nikkor f/2.8G, Nikon) mounted on an 8-megapixel, 14-bit CCD camera (Prosilica GX3300).

\section{Numerics}

The numerical resolution of the Toner-Tu equations Eqs. (1) and (2) were done using a semispectral method with a semi-implicit Euler scheme.

\section{APPENDIX C: LINEAR STABILITY OF THE GENERALIZED TONER-TU EQUATIONS}

In this Appendix, we show how the succession of instabilities of the homogeneous solutions of Eqs. (1) and (2) correctly predict the full phase behavior observed in our experiments and numerical simulations. In the experiments, the flocking transition happens at much lower packing fractions than the solidification, and we thus restrict our analysis to situations where $\rho_{c} \ll \bar{\rho}$. Furthermore, we recall that throughout this article, $\epsilon^{\prime}(\rho)<0$.

\section{Stability of the disordered phases}

We start by considering a homogeneous disordered phase where $\rho=\rho_{0}, W=0$. The linearized dynamics of a small perturbation $\delta X \equiv(\delta \rho, \delta W)$ is given, in Fourier space, by $\delta \dot{X}_{k}=M_{k} \delta X_{k}$, where the dynamical matrix $M_{k}$ is given by

$$
M_{k}=\left(\begin{array}{cc}
-D_{\rho} k^{2} & -i k \\
-\left(\epsilon+\epsilon^{\prime} \rho_{0}\right) i k \sigma & a_{2}\left(\rho_{0}\right)-D_{W} k^{2}
\end{array}\right) .
$$

The eigenvalues $\lambda_{ \pm}$of $M_{k}$ determine the stability of the gas phase. We find

$$
\lambda_{ \pm}=\frac{-\left[\left(D_{\rho}+D_{W}\right) k^{2}-a_{2}\left(\rho_{0}\right)\right] \pm \sqrt{\left[\left(D_{\rho}+D_{W}\right) k^{2}-a_{2}\left(\rho_{0}\right)\right]^{2}-4 k^{2} \sigma\left(\epsilon+\rho_{0} \epsilon^{\prime}\right)-4\left[D_{W} k^{2}-a_{2}\left(\rho_{0}\right)\right] D_{\rho} k^{2}}}{2} .
$$

The gas is therefore unstable when either one of the following conditions is satisfied:

$$
\begin{gathered}
a_{2}\left(\rho_{0}\right)>0 \Leftrightarrow \rho_{0} \epsilon-\rho_{c}>0, \\
\sigma\left(\epsilon+\rho_{0} \epsilon^{\prime}\right)<-\left[D_{W} k^{2}-a_{2}\left(\rho_{0}\right)\right] D_{\rho} .
\end{gathered}
$$

For the case at hand, the second condition is never met in the low-density regime $\rho_{0} \lesssim \rho_{c}$, as $\rho_{c} \ll \bar{\rho}$. In this low-density regime, the first criterion is the standard spinodal instability leading to the emergence of flocking motion [1,43]. As density increases, the system thus undergoes a first linear instability at $\rho_{0} \simeq \rho_{c}$, which corresponds to the transition reported in Figs. 4(b) and 4(c).

At the other end of the density spectrum, deep in the active-solid phase where $\rho_{0} \gg \bar{\rho}$, the criterion Eq. (C3) cannot be realized since $a_{2}(\rho)=-\alpha \rho_{c}<0$. The condition given Eq. (C4) is not realized either because $\epsilon^{\prime} \simeq 0$. As the 
density decreases, both Eqs. (C3) and (C4) could lead to a linear instability of the active solid. Condition Eq. (C3) is realized at the density $\rho_{0}^{*}$ such that $\rho_{0}^{*} \epsilon\left(\rho_{0}^{*}\right)=\rho_{c}$. This condition requires $\epsilon\left(\rho_{0}^{*}\right) \rho_{0}^{*} \ll \rho_{0}^{*}$ and, hence, happens when $\rho_{0} \epsilon\left(\rho_{0}\right)$ is a decreasing function of $\rho_{0}$. Since, for $k=0$, Eq. (C4) reduces to $\left[\rho_{0} \epsilon\left(\rho_{0}\right)\right]^{\prime}<-D_{\rho} \alpha\left(\rho_{c}-\rho_{0} \epsilon\right) / \sigma$, the criterion of Eq. (C4) is already realized at $\rho_{0}^{*}$ : the linear instability of the active solid is always given by Eq. (C4), in which one recognizes the standard form of a MIPS spinodal instability: $\epsilon+\rho \epsilon^{\prime} \ll-K_{1} D_{\rho}$, with $K_{1}$ a positive constant [20]. The linear instability of the active solid leading to the transition illustrated by Figs. 4(e) and 4(f) is thus consistent with the MIPS scenario.

\section{Stability of the polar-liquid phase}

Let us now consider a polar liquid where $\rho=\rho_{0}$, and $W=W_{0}$, with $a_{4} W_{0}^{2}=a_{2}\left(\rho_{0}\right)$. Following the same procedure as above, the linearized dynamics of a small perturbation $\delta X=(\delta \rho, \delta W)$ is defined by the dynamical matrix:

$$
M_{k}=\left(\begin{array}{cc}
M_{\rho \rho} & M_{\rho W} \\
M_{W \rho} & M_{W W}
\end{array}\right)
$$

where

$$
\begin{aligned}
& M_{\rho \rho}(k)=-D_{\rho} k^{2}, \\
& M_{\rho W}(k)=-i k, \\
& M_{W \rho}(k)=-\left(\epsilon+\epsilon^{\prime} \rho_{0}\right)\left(i k \sigma-W_{0} \alpha\right), \\
& M_{W W}(k)=-D_{W} k^{2}-\lambda i k W_{0}-2 a_{4} W_{0}^{2} .
\end{aligned}
$$

The linear stability of the polar liquid is determined by the sign of the real parts of the eigenvalues of $M_{k}, \operatorname{Re}\left[\lambda^{ \pm}(k)\right]$. To be consistent with our modified Toner-Tu equations (1) and (2) that are truncated at the second order in gradients, we only consider $\lambda^{ \pm}(k)$ up to order $k^{2}$. Only $\operatorname{Re}\left[\lambda^{-}(k)\right]$ can become positive and lead to an instability. At order $k^{2}$, it reads:

$$
\begin{aligned}
\operatorname{Re}\left[\lambda^{-}(k)\right]= & -\frac{k^{2}}{8 a_{4}^{3} W_{0}^{4}}\left[\left(\epsilon+\rho \epsilon^{\prime}\right) 2 a_{4} W_{0}^{2}\left(2 a_{4} \sigma+\alpha \lambda\right)\right. \\
& \left.-\alpha^{2}\left(\epsilon+\rho \epsilon^{\prime}\right)^{2}+8 a_{4}^{3} D_{\rho} W_{0}^{4}\right]+o\left(k^{2}\right) .
\end{aligned}
$$

This is a second-order polynomial in $\epsilon+\rho \epsilon^{\prime}$, and the polar liquid is linearly unstable whenever one of the two following conditions is satisfied:

$$
\begin{aligned}
& \epsilon+\rho \epsilon^{\prime}<\frac{a_{4} W_{0}^{2}}{\alpha^{2}}\left(\alpha \lambda+2 a_{4} \sigma\right)\left(1-\sqrt{1+\frac{8 a_{4} D_{\rho} \alpha^{2}}{\left(\alpha \lambda+2 a_{4} \sigma\right)^{2}}}\right), \\
& \epsilon+\rho \epsilon^{\prime}>\frac{a_{4} W_{0}^{2}}{\alpha^{2}}\left(\alpha \lambda+2 a_{4} \sigma\right)\left(1+\sqrt{1+\frac{8 a_{4} D_{\rho} \alpha^{2}}{\left(\alpha \lambda+2 a_{4} \sigma\right)^{2}}}\right) .
\end{aligned}
$$

The first inequality Eq. (C11) can be satisfied only at high densities, when $\rho \sim \bar{\rho}$. It is again of a standard MIPS form, $\epsilon+\rho \epsilon^{\prime}<-W_{0}^{2} K_{2}$, with $K_{2}>0$, and corresponds to the solidification transition from Fig. 4(d) to Fig. 4(e). This criterion defines the spinodal line shown in Movie 8 of Supplemental Material [33] to predict the onset of the active solidification. Both the melting of the active solid and the solidification of the polar liquid thus correspond to standard MIPS instability criteria. It is interesting to note that the local order of the polar liquid merely alters the MIPS instability scenario. Orientational order makes it harder to phase separate through the factor $W_{0}^{2}$ in Eq. (C11). This result is consistent with recent results on the large deviations of active-matter systems that show collective motion to be an optimal strategy to avoid MIPS [54].

On the contrary, the second inequality Eq. $(\mathrm{C} 12)$ is realized at much lower densities, close to $\rho_{0}=\rho_{c}$, when $W_{0}^{2}$ is small enough. It corresponds to the standard linear instability of the polar liquid leading to the formation of Vicsek bands, as shown in Figs. 4(c) and 4(d). Using $W_{0}^{2}=$ $\alpha\left(\rho_{0}-\rho_{c}\right) / a_{4}$ and that $\epsilon=1$ when $\rho_{0} \ll \bar{\rho}$, Eq. (C12) becomes

$\frac{\alpha}{\alpha \lambda+2 a_{4} \sigma}\left(1+\sqrt{1+\frac{8 a_{4} D_{\rho} \alpha^{2}}{\left(\alpha \lambda+2 a_{4} \sigma\right)^{2}}}\right)^{-1}+\rho_{c}<\rho_{0}$.

In the limit of small $D_{\rho}$ relevant for our experiments [34], we recover the usual instability criterion of a homogeneous polar liquid discussed in Ref. [40]:

$$
\rho_{c}+\frac{\alpha}{2 \lambda \alpha+4 a_{4} \sigma}<\rho_{0} .
$$

All in all, we have determined all the spinodal lines governing the two phase transitions found in our experiments:

(i) Flocking transitions between a disordered gas and a polar liquid. The transition from Fig. 4(b) to Fig. 4(c) corresponds to Eq. (C3), and the transition from Fig. 4(d) to Fig. 4(c) corresponds to Eq. (C12).

(ii) MIPS-like transitions between a polar liquid and an active solid. The transition from panels $d$ to $e$ in 
Fig. 4(d) to Fig. 4(e) corresponds to Eq. (C11), and the transition from Fig. 4(f) to Fig. 4(e) corresponds to Eq. (C4).

\section{APPENDIX D: DESCRIPTION OF THE MOVIES}

Movie 1.-Movie 1 shows a polar liquid flowing along a microfluidic racetrack. The dimensions of the observation window are $1.3 \times 1.3 \mathrm{~mm}^{2}$. The movie is slowed down by a factor of 3.8.

Movie 2.-Movie 2 is a close-up in the microfluidic racetrack. We first see the polar-liquid phase and then the compact active solid forming at one end and melting at the other end. The dimensions of the observation window are $1.3 \times 1.3 \mathrm{~mm}^{2}$ wide. The movie is slowed down by a factor of 3.8 .

Movie 3.-Movie 3 shows a typical experiment in a racetrack where an active solid steadily propagates through the homogeneous polar liquid with which it coexists. The movie is sped up by a factor of 30 .

Movie 4.-Movie 4 shows the coarsening dynamics of multiple active solids. The movie is sped up by a factor 30 .

Movie 5.-Hysteresis dynamics upon cycling the magnitude of the electric field. This movie corresponds to the experiments of Figs. 3(e) and 3(f) in the main text. The movie is sped up by a factor of 30 .

Movie 6.-Response to an electric-field quench. The bulk of the active solid is destabilized at all scales. This phenomenon is reminiscent of a spinodal decomposition scenario. The movie is sped up by a factor 30 .

Movie 7.-Emergence of an active solid phase in a circular chamber, response to an electric field ramp. Starting from a polar liquid flowing in the counter clockwise direction a sharp increase of the field result in the emergence of a macroscopic active solid that propagates upstream. The movie is sped up by a factor of 8 . The diameter of the micofluidic chamber is $6 \mathrm{~mm}$.

Movie 8.-Numerical simulations of Eqs (1) and (2) with the same parameters as in Figs. 5(a)-5(c), but with $d x=$ 0.1 and $d t=0.01$ to access longer timescales. Cycling the density $\rho_{0}$ up and down shows a clear hysteresis loop. The polar liquid is stable up to a spinodal density $\rho_{L}^{S}$. After an initial instability, the coarsening process leads the system towards phase separation between a polar liquid at $\rho_{L}^{b}$ and a solid phase, which is propagating backward. When decreasing the density, the solid jam is seen down to $\rho_{0} \simeq \rho_{L}^{b} \ll \rho_{L}^{s}$ highlighting the hysteresis loop.

[1] M. C. Marchetti, J. F. Joanny, S. Ramaswamy, T. B. Liverpool, J. Prost, M. Rao, and R. A. Simha, Hydrodynamics of Soft Active Matter, Rev. Mod. Phys. 85, 1143 (2013).

[2] J. Toner, Y. Tu, and S. Ramaswamy, Hydrodynamics and Phases of Flocks, Ann. Phys. (Amsterdam) 318, 170 (2005).
[3] T. Vicsek and A. Zafeiris, Collective Motion, Phys. Rep. 517, 71 (2012).

[4] A. Cavagna and I. Giardina, Bird Flocks as Condensed Matter, Annu. Rev. Condens. Matter Phys. 5, 183 (2014).

[5] A. Zöttl and H. Stark, Emergent Behavior in Active Colloids, J. Phys. Condens. Matter 28, 253001 (2016).

[6] J. Zhang, E. Luijten, B. A. Grzybowski, and S. Granick, Active Colloids with Collective Mobility Status and Research Opportunities, Chem. Soc. Rev. 46, 5551 (2017).

[7] T. Vicsek, A. Czirók, E. Ben-Jacob, I. Cohen, and O. Shochet, Novel Type of Phase Transition in a System of Self-Driven Particles, Phys. Rev. Lett. 75, 1226 (1995).

[8] V. Schaller, C. Weber, C. Semmrich, E. Frey, and A. R. Bausch, Polar Patterns of Driven Filaments, Nature (London) 467, 73 (2010).

[9] Y. Sumino, K. H. Nagai, Y. Shitaka, D. Tanaka, K. Yoshikawa, H. Chaté, and K. Oiwa, Large-Scale Vortex Lattice Emerging from Collectively Moving Microtubules, Nature (London) 483, 448 (2012).

[10] A. Bricard, J.-B. Caussin, N. Desreumaux, O. Dauchot, and D. Bartolo, Emergence of Macroscopic Directed Motion in Populations of Motile Colloids, Nature (London) 503, 95 (2013).

[11] J. Deseigne, O. Dauchot, and H. Chaté, Collective Motion of Vibrated Polar Disks, Phys. Rev. Lett. 105, 098001 (2010).

[12] N. Kumar, H. Soni, S. Ramaswamy, and A. K. Sood, Flocking at a Distance in Active Granular Matter, Nat. Commun. 5, 4688 (2014).

[13] G. Vásárhelyi, C. Virágh, G. Somorjai, T. Nepusz, A. E. Eiben, and T. Vicsek, Optimized Flocking of Autonomous Drones in Confined Environments, Sci. Robotics 3, eaat3536 (2018).

[14] F. D. C. Farrell, M. C. Marchetti, D. Marenduzzo, and J. Tailleur, Pattern Formation in Self-Propelled Particles with Density-Dependent Motility, Phys. Rev. Lett. 108, 248101 (2012).

[15] G. Grégoire and H. Chaté, Onset of Collective and Cohesive Motion, Phys. Rev. Lett. 92, 025702 (2004).

[16] F. Peruani, T. Klauss, A. Deutsch, and A. Voss-Boehme, Traffic Jams, Gliders, and Bands in the Quest for Collective Motion of Self-Propelled Particles, Phys. Rev. Lett. 106, 128101 (2011).

[17] F. Peruani and M. Baer, A Kinetic Model and Scaling Properties of Non-Equilibrium Clustering of Self-Propelled Particles, New J. Phys. 15, 065009 (2013).

[18] D. Nesbitt, G. Pruessner, and C. F. Lee, Using a Lattice Boltzmann Method to Uncover Novel Phase Transitions in Dry Polar Active Fluids, arXiv:1902.00530.

[19] E. Sese-Sansa, I. Pagonabarraga, and D. Levis, Velocity Alignment Promotes Motility-Induced Phase Separation, Europhys. Lett. 124, 30004 (2018).

[20] M. E. Cates and J. Tailleur, Motility-Induced Phase Separation, Annu. Rev. Condens. Matter Phys. 6, 219 (2015).

[21] Y. Fily and M. C. Marchetti, Athermal Phase Separation of Self-Propelled Particles with No Alignment, Phys. Rev. Lett. 108, 235702 (2012).

[22] I. Buttinoni, J. Bialké, F. Kümmel, H. Löwen, C. Bechinger, and T. Speck, Dynamical Clustering and Phase Separation in Suspensions of Self-Propelled Colloidal Particles, Phys. Rev. Lett. 110, 238301 (2013). 
[23] G. S. Redner, M. F. Hagan, and A. Baskaran, Structure and Dynamics of a Phase-Separating Active Colloidal Fluid, Phys. Rev. Lett. 110, 055701 (2013).

[24] J. Bialké, H. Löwen, and T. Speck, Microscopic Theory for the Phase Separation of Self-Propelled Repulsive Disks, Europhys. Lett. 103, 30008 (2013).

[25] I. Theurkauff, C. Cottin-Bizonne, J. Palacci, C. Ybert, and L. Bocquet, Dynamic Clustering in Active Colloidal Suspensions with Chemical Signaling, Phys. Rev. Lett. 108, 268303 (2012).

[26] G. Liu, A. Patch, F. Bahar, D. Yllanes, R. D. Welch, M. C. Marchetti, S. Thutupalli, and J. W. Shaevitz, A MotilityInduced Phase Transition Drives Myxococcus xanthus Aggregation, Phys. Rev. Lett. 122, 248102 (2019).

[27] M. N. van der Linden, L. C. Alexander, D. G. A. L. Aarts, and O. Dauchot, Interrupted Motility Induced Phase Separation in Aligning Active Colloids, arXiv:1902.08094.

[28] L. Caprini, U. M. B. Marconi, and A. Puglisi, Activity Induced Delocalization and Freezing in Self-Propelled Systems, Sci. Rep. 9, 1386 (2019).

[29] G. Quincke, Uber Rotationen im Constanten Electrischen Felde, Ann. Phys. Chem. 59, 417 (1896).

[30] H. Chaté, F. Ginelli, G. Grégoire, and F. Raynaud, Collective Motion of Self-Propelled Particles Interacting without Cohesion, Phys. Rev. E 77, 046113 (2008).

[31] E. Bertin, M. Droz, and G. Grégoire, Hydrodynamic Equations for Self-Propelled Particles: Microscopic Derivation and Stability Analysis, J. Phys. A 42, 445001 (2009).

[32] A. P. Solon, H. Chaté, and J. Tailleur, From Phase to Microphase Separation in Flocking Models: The Essential Role of Nonequilibrium Fluctuations, Phys. Rev. Lett. 114, 068101 (2015).

[33] See Supplemental Material at http://link.aps.org/ supplemental/10.1103/PhysRevX.9.031043 for eight Supplemental Movies illustrate our experimental findings. See also Appendix D for a description.

[34] D. Geyer, A. Morin, and D. Bartolo, Sounds and Hydrodynamics of Polar Active Fluids, Nat. Mater. 17, 789 (2018).

[35] G. Briand and O. Dauchot, Crystallization of Self-Propelled Hard Discs, Phys. Rev. Lett. 117, 098004 (2016).

[36] A. Bricard, J.-B. Caussin, D. Das, C. Savoie, V. Chikkadi, K. Shitara, O. Chepizhko, F. Peruani, D. Saintillan, and D. Bartolo, Emergent Vortices in Populations of Colloidal Rollers, Nat. Commun. 6, 7470 (2015).

[37] A. Morin, N. Desreumaux, J.-B. Caussin, and D. Bartolo, Distortion and Destruction of Colloidal Flocks in Disordered Environments, Nat. Phys. 13, 63 (2017).

[38] A. Morin, D. L. Cardozo, V. Chikkadi, and D. Bartolo, Diffusion, Subdiffusion, and Localization of Active Colloids in Random Post Lattices, Phys. Rev. E 96, 042611 (2017).

[39] J.-B. Caussin, A. Solon, A. Peshkov, H. Chaté, T. Dauxois, J. Tailleur, V. Vitelli, and D. Bartolo, Emergent Spatial
Structures in Flocking Models: A Dynamical System Insight, Phys. Rev. Lett. 112, 148102 (2014).

[40] A. P. Solon, J.-B. Caussin, D. Bartolo, H. Chaté, and J. Tailleur, Pattern Formation in Flocking Models: A Hydrodynamic Description, Phys. Rev. E 92, 062111 (2015).

[41] A. P. Solon, J. Stenhammar, R. Wittkowski, M. Kardar, Y. Kafri, M.E. Cates, and J. Tailleur, Pressure and Phase Equilibria in Interacting Active Brownian Spheres, Phys. Rev. Lett. 114, 198301 (2015).

[42] A. P. Solon, J. Stenhammar, M. E. Cates, Y. Kafri, and J. Tailleur, Generalized Thermodynamics of MotilityInduced Phase Separation: Phase Equilibria, Laplace Pressure, and Change of Ensembles, New J. Phys. 20, 075001 (2018).

[43] S. Mishra, A. Baskaran, and M. C. Marchetti, Fluctuations and Pattern Formation in Self-Propelled Particles, Phys. Rev. E 81, 061916 (2010).

[44] A. G. Thompson, J. Tailleur, M. E. Cates, and R. A. Blythe, Lattice Models of Nonequilibrium Bacterial Dynamics, J. Stat. Mech. (2011) P02029 2029.

[45] J. Stenhammar, A. Tiribocchi, R. J. Allen, D. Marenduzzo, and M. E. Cates, Continuum Theory of Phase Separation Kinetics for Active Brownian Particles, Phys. Rev. Lett. 111, 145702 (2013).

[46] G. Gonnella, D. Marenduzzo, A. Suma, and A. Tiribocchi, Motility-Induced Phase Separation and Coarsening in Active Matter, C.R. Phys. 16, 316 (2015).

[47] A. P. Solon, M. E. Cates, and J. Tailleur, Active Brownian Particles and Run-and-Tumble Particles: A Comparative Study, Eur. Phys. J. Spec. Top. 224, 1231 (2015).

[48] E. Tjhung, C. Nardini, and M. E. Cates, Cluster Phases and Bubbly Phase Separation in Active Fluids: Reversal of the Ostwald Process, Phys. Rev. X 8, 031080 (2018).

[49] J. R. Melcher and G. I. Taylor, Electrohydrodynamics: A Review of the Role of Interfacial Shear Stresses, Annu. Rev. Fluid Mech. 1, 111 (1969).

[50] S. Kim and S. J. Karrila, Microhydrodynamics: Principles and Selected Applications (Butterworth-Heinemann, Boston, 2013).

[51] D. Blair and E. Dufresne, MATLAB particle tracking code repository, http://site.physics.georgetown.edu/matlab/index .html.

[52] J. C. Crocker and D. G. Grier, Methods of Digital Video Microscopy for Colloidal Studies, J. Colloid Interface Sci. 179, 298 (1996).

[53] P. J. Lu, P. A. Sims, H. Oki, J. B. Macarthur, and D. A. Weitz, Target-Locking Acquisition with Real-Time Confocal (TARC) Microscopy, Opt. Express 15, 8702 (2007).

[54] T. Nemoto, É. Fodor, M. E. Cates, R. L. Jack, and J. Tailleur, Optimizing Active Work: Dynamical Phase Transitions, Collective Motion, and Jamming, Phys. Rev. E 99, 022605 (2019). 